\title{
Light-Scattering Properties of Representative, Morphological Rutile Titania Particles Studied Using a Finite-Element Method
}

\author{
Erik S. Thiele* and Roger H. French ${ }^{\star}$ \\ DuPont Company Central Research, E356-384 Experimental Station, Wilmington, Delaware 19880
}

The light-scattering performance of $\mathrm{TiO}_{2}$ pigment depends intimately upon particle size, size distribution, shape and dispersion quality. $\mathrm{TiO}_{2}$ particles exhibit complex shapes and have anisotropic optical constants, and particulate dispersions of $\mathrm{TiO}_{2}$ are characterized by complicated microstructures such as aggregates and agglomerates. Despite this complexity, the theoretical understanding of light scattering by white pigment has been based upon Mie theory, which is restricted to the case of a single, optically isotropic sphere. We utilize a finite-element method which produces rigorous solutions to Maxwell's equations to determine computationally the light-scattering properties of complex particulate microstructures. This represents a significant step beyond the restrictions of Mie theory, providing a method to determine quantitatively the effects of particle shape, optical anisotropy, and interactions between neighboring particles upon the light-scattering properties of white pigments in coatings. In the present study, we use the finite-element method first to compute the light-scattering properties of a single, morphological rutile particle with a representative size and shape. These results are compared to the light-scattering properties of the optically isotropic, equivalent volume sphere using Mie theory. Neither the average index nor the weighted sum approximation offers clear advantages in this case. Second, the far-field lightscattering properties of two such particles interacting at near field are determined as a function of the interparticle separation. The agglomerated pair of particles exhibits a $20 \%$ decrease relative to the single particle in the scattering parameter associated with the hiding power of a paint film. The basis for this decrease is the same as for the crowding effect observed in extensive paint films. The results of both sets of computations are compared to Mie theory to determine the sizes of spherical particles with equivalent scattering cross sections. These comparisons highlight the inherent difficulties in using Mie theory to evaluate particle size by light-scattering methods.

\section{Introduction}

$\mathrm{R}$ UTILE titania offers an attractive combination of physical properties which has made it the most widely used white pigment in the worldwide marketplace. ${ }^{1,2}$ A fundamental goal of both manufacturers and consumers of rutile titania pigment is to maximize the light-scattering efficiency of the pigment in end use. The achievement of this goal requires quantitatively identifying, and subsequently controlling, the factors which

V. Tikare — contributing editor

\footnotetext{
Manuscript No. 190860. Received July 7, 1997; approved December 3, 1997. Presented at the 99th Annual Meeting of The American Ceramic Society, Cincin nati, OH, May 5-7, 1997 (Theory and Computational Modeling Symposium, Paper No. SXIX-044-97)

Member, American Ceramic Society.
}

impact the light-scattering efficiency of rutile titania powders. This light-scattering efficiency is controlled by a number of factors, including the particle size distribution, the degree of particle agglomeration, and the degree of near-field optical interaction between neighboring particles in a pigmented microstructure. However, the quantitative influence of each of these factors upon the hiding power of rutile titania is not fully understood, due in large part to the difficulty of controlling laboratory experiments to the degree that the effect of each can be studied independently.,4

Due to the inherent experimental challenges, theoretical modeling attempts have been made to understand lightscattering properties of rutile titania. These efforts have typically relied upon Mie theory, which provides an exact treatment of the light-scattering properties of a single, optically isotropic sphere. ${ }^{5-7}$ Examples of such studies include investigation of the theoretical hiding power of rutile titania versus that of air voids, ${ }^{8}$ the crowding effect in highly loaded films ${ }^{9-12}$ and the application of a multiple-scattering model to predict the light-scattering efficiency of paint films. ${ }^{13}$ In reality, individual rutile titania particles exhibit both optical anisotropy and complex shapes. In addition, pigmented films typically contain multiple-particle aggregates in which near-field optical interactions between neighboring particles play a critical role. Mie theory is therefore not capable of rigorously describing the light-scattering properties of realistic rutile titania particle shapes and microstructures. A high-resolution scanning electron micrograph of a commercial rutile titania pigment powder is shown in Fig. 1. Both the characteristic, nonspherical shape of the primary particles and the possibility of particle aggregation are highlighted in the micrograph.

To move beyond the limitations of Mie theory, we employ a finite-element method which produces rigorous solutions to Maxwell's equations for electromagnetic radiation interacting with arbitrary microstructures. The finite-element method permits computation of the light-scattering properties of realisti-

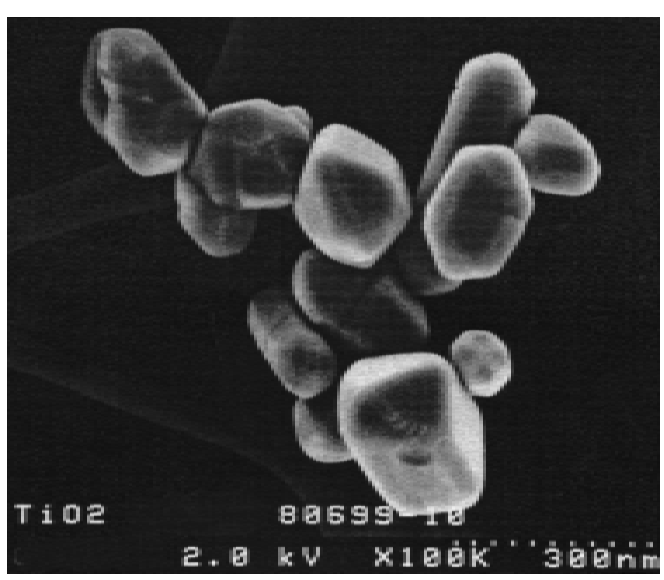

Fig. 1. High-resolution scanning electron micrograph of rutile $\mathrm{TiO}_{2}$ particles, showing their characteristic morphological shapes. 
cally shaped rutile titania particles and the effects of optical interactions between neighboring particles in a pigmented film. Importantly, the finite-element approach can be used to determine the light-scattering properties of realistic systems which would otherwise be inaccessible. Following a description of the finite-element approach, several illustrative case studies are presented below, with comparison between the results of Mie theory and the finite-element method provided where applicable.

\section{Methods}

\section{(1) Finite-Element Approach}

In the finite-element approach to electromagnetic radiation scattering, a combined form of Maxwell's equations is solved for electric field throughout a computational volume $V_{0}$ which has been discretized into finite-volume elements. ${ }^{14}$ In the absence of free charges, the time-domain point form of electric field derived from Maxwell's equations is

$$
\varepsilon \frac{\partial^{2} \vec{E}}{\partial t^{2}}+\sigma \frac{\partial \vec{E}}{\partial t}=-\frac{1}{\mu} \nabla \times \nabla \times \vec{E}
$$

In Eq. (1), $E$ is electric field amplitude, $\varepsilon$ is the permittivity of the medium, $\sigma$ is the conductivity of the medium, and $\mu$ is the permeability of the medium. The inner dot product of this governing differential equation is formed with the arbitrary vector weighting function $G$, which is a function of both time and position, and integrated over each volume element in the model

$$
\begin{aligned}
\iint_{V_{0}} \int_{\vec{G}} \overrightarrow{(\varepsilon} \cdot\left(\varepsilon \frac{\partial^{2} \vec{E}}{\partial t^{2}}+\sigma \frac{\partial \vec{E}}{\partial t}\right) \mathrm{d} V_{0}= & -\frac{1}{\mu} \iint_{V_{0}} \int \\
& \vec{G} \cdot(\nabla \times \nabla \times \vec{E}) \mathrm{d} V_{0}
\end{aligned}
$$

Electric field values are defined at the nodes of the finiteelement mesh, and fields are interpolated within each volume element using a linear shape function. In the finite-element implementation used in the present research, the weighting function is chosen to have the same spatial variation as the shape function (the Galerkin formulation), yielding a symmetric system of linear equations in the unknown electric field. ${ }^{15}$ The second temporal derivative appearing in Eqs. (1) and (2) is approximated using an explicit scheme based upon the central difference approximation

$$
\frac{\partial^{2} \vec{E}(t)}{\partial t^{2}} \approx \frac{\vec{E}(t+\Delta t)-2 \vec{E}(t)+\vec{E}(t-\Delta t)}{(\Delta t)^{2}}
$$

where $\Delta t$ is the time step used in the computation. The timedomain finite-element software used in the present research has been applied by other investigators to a number of systems of practical interest, including the determination of lightscattering properties of features on silicon wafers ${ }^{16}$ and evaluation of the performance of integrated optical devices ${ }^{17}$ and waveguides. ${ }^{18-20}$

The finite-element models in the present study are constructed in Cartesian coordinate systems by specifying edge dimensions, the finite-element mesh density, particle position(s) and shape(s), and the optical properties of the constituent materials at the wavelength of interest. Typical models contain 1-2 million elements. Each individual computation employs a single wavelength of electromagnetic radiation which propagates in the model as a polarized plane wave with a specified Poynting vector. The electromagnetic radiation is allowed to propagate in the finite-element model in the time domain, with electric field amplitude and phase computed at each node in the finite-element model per time step. A suffi- cient number of time steps are employed in a computation to ensure that steady state is achieved; steady state is confirmed by reviewing time histories of electric field amplitude at selected nodes in the model to verify convergence. The near-field scattered electric field amplitudes are extrapolated to far field by positioning a Kirchoff surface located a few elements inside the walls of the finite-element model, on which equivalent electric and magnetic currents are computed from the tangential components of scattered electric and magnetic field. From these equivalent currents the scattered electric field at near field is extrapolated onto the surface of a far-field sphere having a radius which is much greater than the wavelength of light using the equivalence theorem. ${ }^{21}$ Extrapolation eliminates evanescent electric field components which are present at near field so that only propagating electromagnetic waves contribute to the far-field result. The simulation of diffuse illumination is accomplished by superimposing the far-field scattered intensities computed in a series of individual computations for which the illumination directions have been varied stepwise over the full range of possible orientations. The platform used in these computations is a Cray C-94 supercomputer.

Once extrapolation of scattered electric field and intensities $I$ has been accomplished, it is possible to extract macroscopically observable physical quantities such as the scattering cross section $C_{\mathrm{sca}}$ of the microstructure being modeled, and the angular distribution of scattered light. The scattering cross section is defined in Eq. (4) and is expressed in units of area.

$$
C_{\text {sca }}=\frac{\iint_{4 \pi} I_{\text {sca }} \mathrm{d} \Omega}{I_{0}}
$$

where $I_{0}$ is the incident intensity. It is useful to normalize the scattering cross section by the volume $V$ of the scattering material in the finite-element model. The scattering coefficient $S$ is defined in Eq. (5) and is expressed in units of inverse length.

$$
S=\frac{C_{\text {sca }}}{V}
$$

The scattering coefficient provides information about the strength of light scattering by a particular microstructure on a per-volume basis. It provides no direct information, however, about the efficiency of a microstructure in deflecting light from the incident direction. In a paint film, for example, a combination of strong scattering per volume and strong deflection of light is desired, since it is this combination that imparts hiding power to a paint film. The asymmetry parameter $g$ (Eq. (6)) is the average cosine of the scattering angle associated with a scattering microstructure, weighted by the scattered intensity as a function of angle.

$$
g=\frac{\int_{4 \pi} \int_{4 \pi} I(\theta) \cos \theta d \theta}{\iint I(\theta) d \theta}
$$

The asymmetry parameter $g$ is unitless and varies between -1 (for perfect backward scattering) and 1 (for perfect forward scattering). Both the asymmetry parameter and the scattering coefficient play prominent roles in multiple-scattering theory, where the term defined here as the angle-weighted scattering coefficient $\sigma$ (Eq. (7)) expresses the combined ability of a scattering feature to strongly deflect incident electromagnetic radiation off the incident direction. ${ }^{22}$

$$
\sigma=S(1-g)
$$

The term $\sigma$, like the scattering coefficient $S$, is expressed in units of inverse length. It can be considered a definitive figure of merit in the context of the ability of a scattering feature in a paint film to contribute to hiding power. Throughout this study, 
scattering results are expressed in terms of both the scattering coefficient $S$ and the angle-weighted scattering coefficient $\sigma$.

\section{(2) Mie Theory}

To quantify the accuracy of the finite-element method, it is useful to compare results obtained using this method for scattering by an isotropic sphere to the exact results obtained using Mie theory. Mie theory has been described in full detail previously. ${ }^{5-7}$ To compute the Mie theory results presented below, we have employed the computer code provided by Bohren and Huffman, ${ }^{7}$ with modifications to allow computation of the asymmetry parameter. In this computational approach, series expansions are performed to compute the scattering cross section at far field.

As stated above, it is not possible to apply Mie theory directly to the problem of light scattering by a rutile titania sphere, since Mie theory is limited to the case of an optically isotropic sphere and rutile titania is optically anisotropic. It is therefore necessary to treat rutile titania in an approximate manner. Rutile titania is a uniaxial crystal, exhibiting the higher, extraordinary refractive index for light polarized parallel to the $c$-axis of the crystal and the lower, ordinary refractive index for light polarized parallel to either the $a$ - or $b$-axis. Two methods for approximating rutile as an isotropic material have been proposed in the literature. One approach has been to compute the mean of the refractive indexes along the three crystal axes at the light wavelength of interest ${ }^{13}$ and subsequently apply Mie theory to compute far-field scattering parameters. We refer to this as the average index approximation. Another method has been to compute separately the far-field scattering parameters for an isotropic sphere having the ordinary and extraordinary refractive indexes of rutile titania and subsequently compute the weighted sum of these separate farfield results. 8,13 The weighted sum consists of two-thirds the result for the ordinary refractive index plus one-third the result for the extraordinary refractive index. We refer to this as the weighted sum approximation. Both of these approximate approaches are used in the present study in comparing far-field results obtained using the finite-element method to results obtained using Mie theory.

The wavelength of interest for tint strength tests of paint films is $560 \mathrm{~nm}$, the center of the visible spectrum. This wavelength is used in the computations performed throughout this study. At $560 \mathrm{~nm}$, the ordinary refractive index of rutile titania is 2.64 , while the extraordinary refractive index is $2.94 .^{23}$ The average refractive index along the three crystal axes of rutile at $560 \mathrm{~nm}$ is therefore 2.74 . The case of rutile titania embedded in a typical acrylic resin ${ }^{8}$ can be represented using Mie theory and

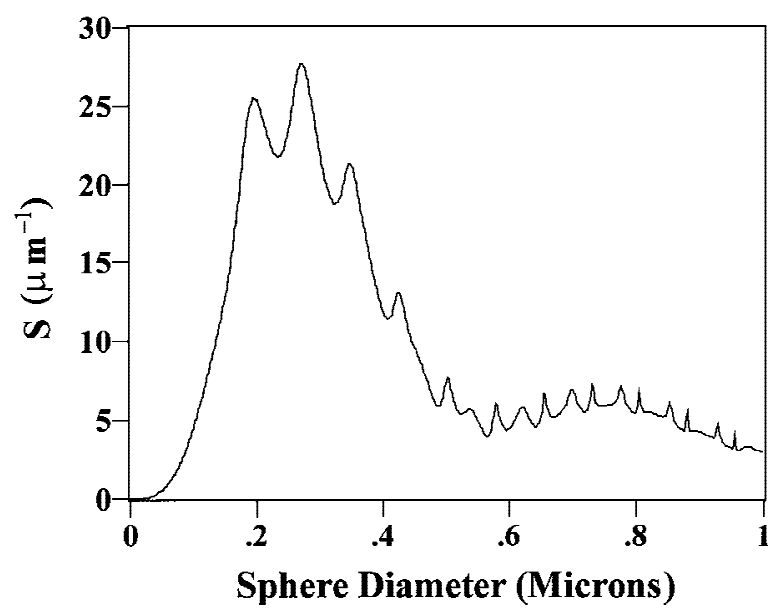

Fig. 2. Scattering coefficient $S$ versus sphere diameter for an optically isotropic sphere from Mie theory, using the average index approximation.

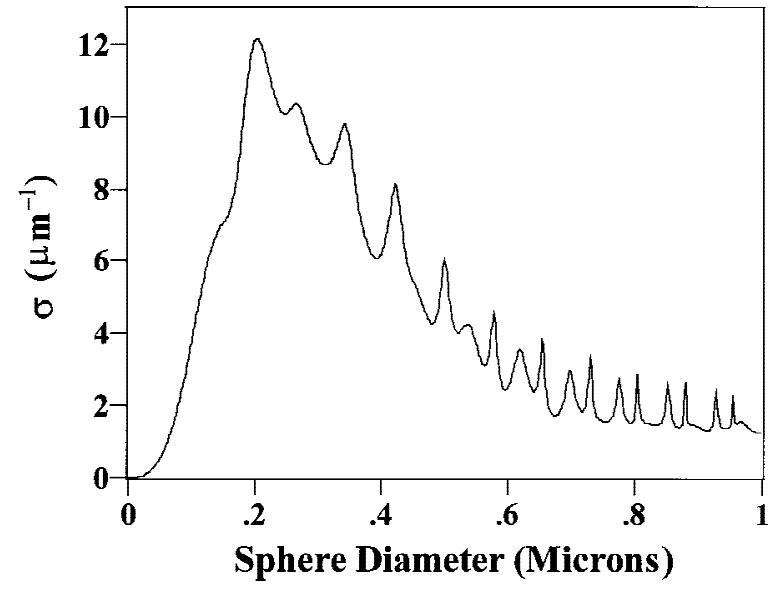

Fig. 3. Angle-weighted scattering coefficient $\sigma$ versus sphere diameter for an optically isotropic sphere from Mie theory, using the average index approximation.

the average index approximation by computing the lightscattering behavior of an isotropic sphere with refractive index $n=2.74$ embedded in a medium with $n=1.514$. For these conditions, the scattering coefficient $S\left(\mu \mathrm{m}^{-1}\right)$ is shown in Fig. 2 as a function of sphere diameter. The curve can be considered a rolling curve which is punctuated by resonant peaks. The greatest $S$ values occur for sphere diameters in the range 0.18 $0.30 \mu \mathrm{m}$, with a maximum value of $S=27.74 \mu \mathrm{m}^{-1}$ for the diameter $0.272 \mu \mathrm{m}$. Likewise, the angle-weighted scattering coefficient $\sigma\left(\mu \mathrm{m}^{-1}\right)$ for these same conditions is shown as a function of sphere diameter in Figure 3. The maximum value of the angle-weighted scattering coefficient $\sigma=12.17 \mu \mathrm{m}^{-1} \mathrm{oc}-$ curs for the sphere diameter $0.204 \mu \mathrm{m}$.

It is of interest to compare the results of Mie theory calculations under the weighted sum approximation to those of the average index approximation discussed above. The result of computing the weighted sum of the separate scattering coefficient $S$ curves for $n=2.94$ and $n=2.64$ spheres embedded in an $n=1.514$ medium is shown in Fig. 4 as a function of sphere diameter. This result exhibits less pronounced resonant peaks in $S$ than the analogous plot generated using the average index approximation shown in Fig. 2. This is expected, since the resonant peaks in the two individual curves used to form the weighted sum are offset from one another. The maximum $S$ values for the weighted sum approximation occur in the range

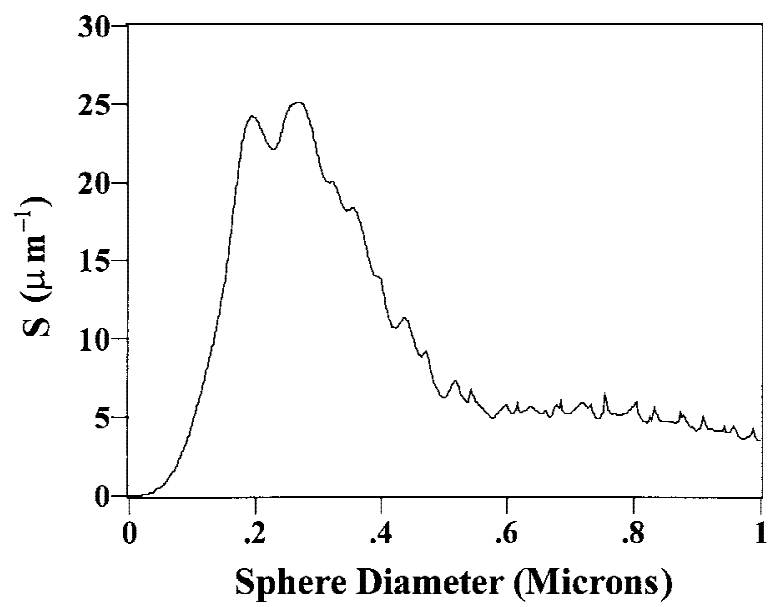

Fig. 4. Scattering coefficient $S$ versus sphere diameter for an optically isotropic sphere from Mie theory, using the weighted sum approximation. 
0.18 to $0.3 \mu \mathrm{m}$, as with the average index approximation. The maximum value $S=25.08 \mu \mathrm{m}^{-1}$ occurs for the diameter 0.270 $\mu \mathrm{m}$, about $10 \%$ lower than the maximum value $S=27.74$ $\mu \mathrm{m}^{-1}$ observed in the case of the average index approximation shown in Fig. 2. The angle-weighted scattering coefficient $\sigma$ $\left(\mu \mathrm{m}^{-1}\right)$ is shown in Fig. 5 as a function of sphere diameter using the weighted sum approximation. This curve is in close agreement with that computed using the average index approximation, shown in Fig. 3. In the case of the weighted sum approximation, the maximum value of the angle-weighted scat-

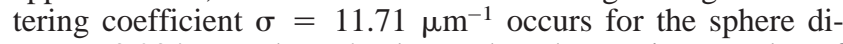
ameter $0.204 \mu \mathrm{m}$, about $4 \%$ lower than the maximum value of the angle-weighted scattering coefficient $\sigma=12.17 \mu \mathrm{m}^{-1} \mathrm{ob}-$ served in the case of the average index approximation.

The average index approximation offers the advantage of explicitly defining a single refractive index for the material, therefore requiring only a single set of computations to obtain far-field results. The weighted sum approximation offers the advantage of smoothing out the sharp resonant peaks associated with particular sphere diameters in Mie theory computations, artifacts of the high symmetry of a sphere which in practice are not observed in scattering by real systems of multiple particles.

\section{(3) Accuracy of the Finite-Element Method}

The analytical results of Mie theory provide a basis with which to compare the accuracy of finite-element computations for the case of an isotropic sphere. This comparison is essential to determine the expected accuracy for computations for which no analytical solutions exist. Figure 6 shows the results of a finite-element computation performed for the case of scattering of 560-nm light by $\alpha$ 200-nm sphere. The sphere has been assigned the refractive index $n=2.74$ using the average index approximation and is surrounded by a medium of refractive index $n=1.51$, an approximation of a rutile particle in a polymeric resin. This model contains $\sim 1.3$ million cubic elements, $3.6 \mathrm{~nm}$ on edge. The upper left panel of Fig. 6 shows a three-dimensional view of the sphere with the surrounding medium hidden; the cubic elements which compose the sphere are visible. The upper right panel of Fig. 6 shows a cross-sectional view of the finite-element model, including the sphere and the surrounding medium. In this computation, light propagates in the $+x$ direction, from top to bottom in the upper right panel of Fig. 6, with incident intensity equal to unity. The lower two panels of Fig. 6 show scattered intensities in the geometric domains displayed in the corresponding upper panels. The scat-

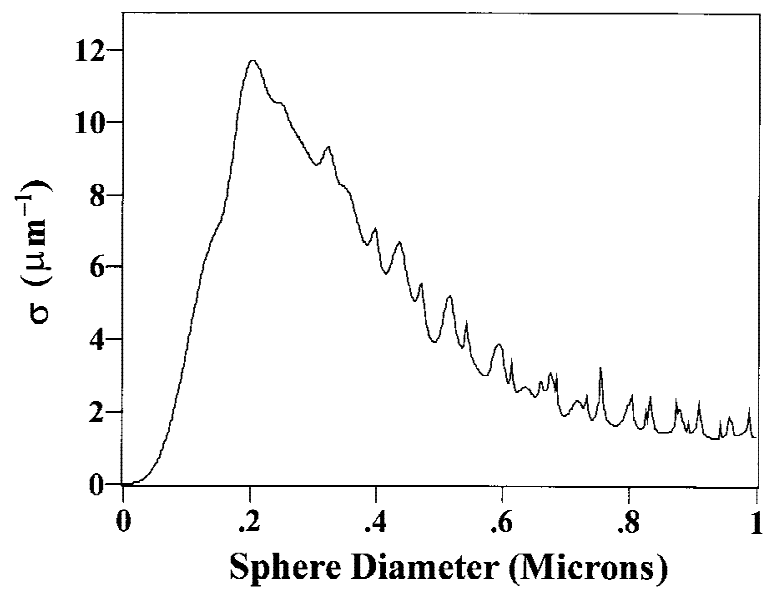

Fig. 5. Angle-weighted scattering coefficient $\sigma$ versus sphere diameter for an optically isotropic sphere from Mie theory, using the weighted sum approximation. tered intensities exceed the unit illumination intensity by a factor of 4 in the lower two panels of Fig. 6. This is a characteristic of particles which are highly resonant with the incident radiation. Such particles can exhibit scattering cross sections which are several times larger than their geometric cross sections.

In order to extract far-field scattering parameters from nearfield results, the near-field results are extrapolated onto the surface of a far-field sphere. This extrapolation has the effect of separating propagating components of the near-field scattered light from the nonpropagating, evanescent components. Figure 7 shows the scattered intensity on the surface of a far-field sphere with a radius $500 \mu \mathrm{m}$. The scattered intensity on the surface of this sphere can be integrated to compute the scattering cross section for the sphere shown in Fig. 6. Using the appropriate weighting, the far-field intensity can also be integrated to compute the asymmetry parameter (Eq. (6)). The scattering cross section computed for the finite-element model shown in Fig. 6 is $0.1027 \mu \mathrm{m}^{2}$, compared to the exact result of $0.1021 \mu \mathrm{m}^{2}$ from Mie theory, indicating an error of $0.55 \%$ for the finite-element method.

We have computed the scattering cross section for the case of the optically isotropic 200-nm sphere with $n=2.74$ embedded in a medium with $n=1.514$ using a range of finiteelement mesh densities. This sphere is highly resonant with the incident radiation and therefore presents a challenge to the ability of the finite-element method to resolve electric field gradients at the surface of the sphere. The finite-element density $(\rho)$ is defined in terms of the light wavelength $(\lambda)$ divided by the refractive index of the highest index material $\left(n_{\text {max. }}\right)$ in the finite-element model, divided by the finite-element edge length $(l)$.

$$
\rho=\frac{\lambda}{n_{\text {max. }} l}
$$

This dimensionless ratio represents the number of elements per compressed light wavelength in the highest refractive index material in a finite-element model. It reflects the ability of the finite-element mesh to resolve gradients in electric field, with higher values implying better resolution of gradients. A plot of error $(\%)$ of the finite-element calculation versus Mie theory as a function of $\rho$ resulting from this study is shown in Fig. 8. The error varies systematically between $8 \%$ for the case $\rho=10$ and $0.55 \%$ for the case $\rho=68$. These values provide guidance in estimating the errors associated with the finite-element computations on systems for which exact solutions may not exist for comparison. For typical finite-element models containing a single particle, values of $\rho>40$ are used, suggesting that error levels are confined to $\sim 2 \%$.

\section{Scattering by a Morphological Rutile Particle}

The real utility of the finite-element method lies in the capability to determine the light-scattering properties of realistic microstructures which are more complex than the case of a single sphere. We have applied the finite-element method to the case of 560-nm light scattering by a single, morphological rutile titania particle. This particle, shown in the upper panels of Fig. 9, has a width $0.175 \mu \mathrm{m}$ and a tip-to-tip length of 0.35 $\mu \mathrm{m}$. The shape and size of this particle are representative of rutile titania pigment and have been chosen to illustrate the scattering properties of a typical pigment particle. In contrast to the finite-element computations performed for the case of a single sphere in Section III of this study, this rutile particle has been assigned the appropriate anisotropic optical constants. As in the calculations described above, the particle is surrounded by a medium with refractive index $n=1.514$, representative of an acrylic resin. This model contains $\sim 1.6$ million cubic elements, $3.6 \mathrm{~nm}$ on edge. This corresponds to a finite-element mesh density of $\rho=50$ elements per light wavelength in the model. The upper left panel of Fig. 9 shows a three- 


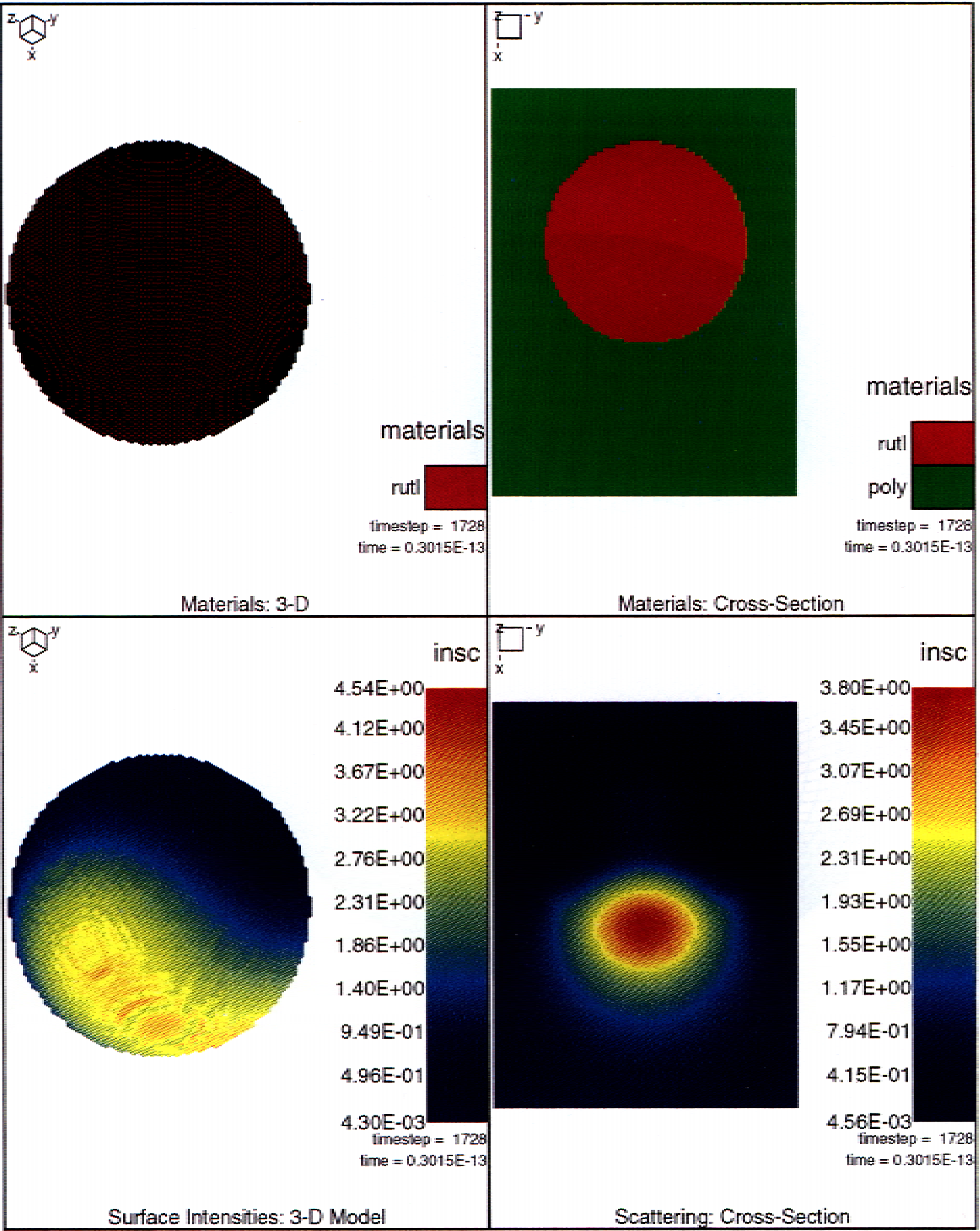

Fig. 6. Finite-element model and near-field scattering results for a 200 -nm-diameter, $n=2.74$ sphere in $n=1.514$ resin; the light wavelength is $560 \mathrm{~nm}$. On the left are three-dimensional views of the model and scattered light intensity, while on the right are cross-sectional views of the model and scattered-light intensity.

dimensional view of the sphere with the surrounding medium hidden; the cubic elements which compose the particle are visible. The upper right panel shows a cross-sectional view of the finite-element model, including the particle and the surrounding medium. In this case, light is propagating in the finite-element model with components in both the $+x$ and $+z$ directions with unit incident intensity. The lower two panels of Fig. 9 show scattered intensities in the geometric domains displayed in the corresponding upper panels. As in the case of the spherical particle shown in Fig. 6, scattered intensities in the near field are several times greater than the incident intensity.

In general, the rutile titania particles in a paint film are 


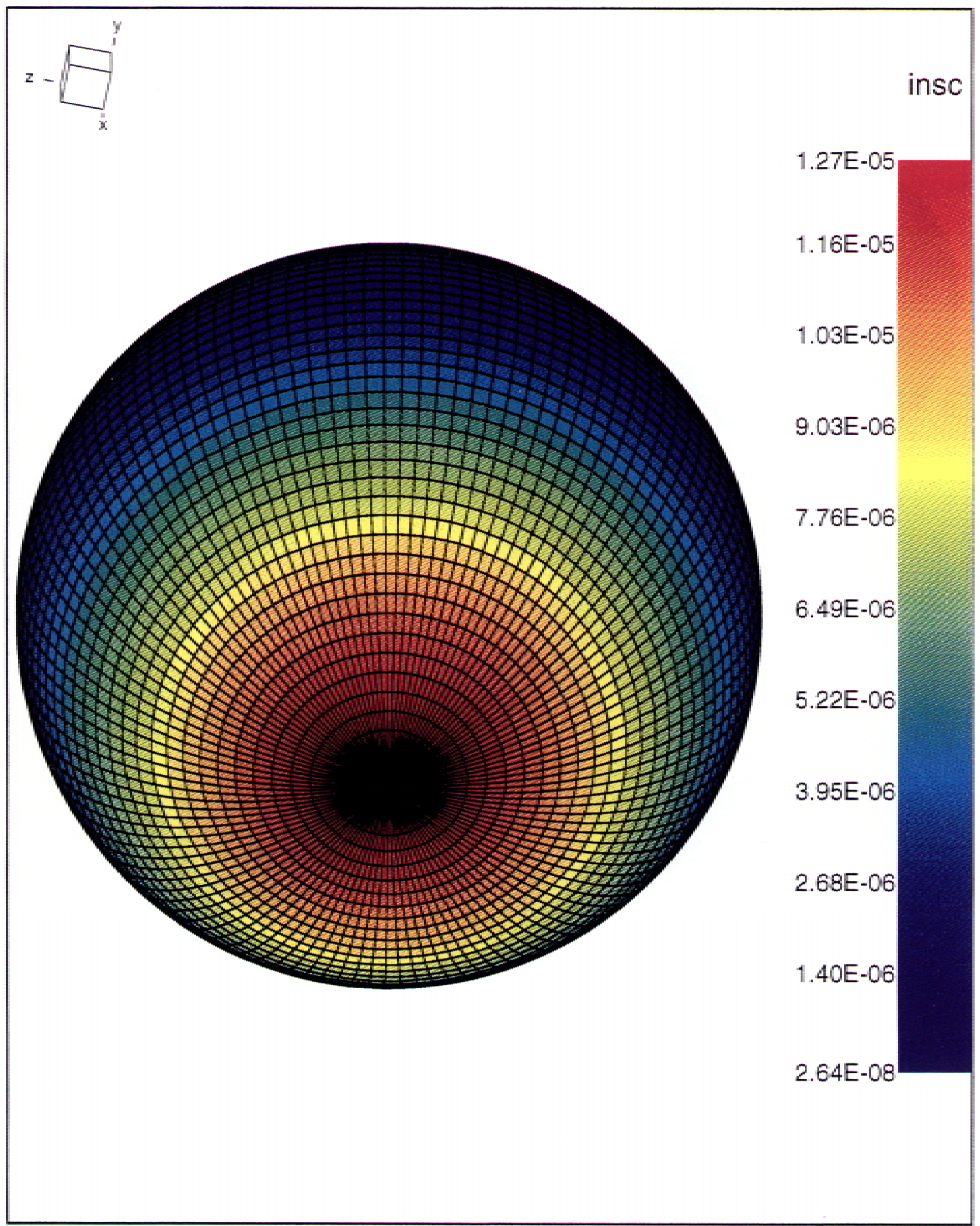

Fig. 7. Scattered intensity on the surface of a far-field sphere with radius of $500 \mu \mathrm{m}$ for a 200 -nm-diameter, $n=2.74$ sphere in $n=1.514$ resin; the light wavelength is $560 \mathrm{~nm}$.

randomly oriented. In order to determine the light-scattering properties of the morphological, anisotropic rutile particle shown in Fig. 9 under conditions of diffuse illumination, a set of individual finite-element computations with different angles of light incidence are performed. Two individual plane waves having mutually perpendicular polarizations are used for each illumination direction. To determine far-field scattering prop- erties for the case of random illumination, far-field intensities for the individual illuminations are superimposed. The presence of several symmetry mirror planes in the geometry of the rutile particle shown in Fig. 9 requires that only one half-octant of the particle be illuminated to determine the full lightscattering properties of the particle. In the present case, 15 different illumination directions were employed over one half- 


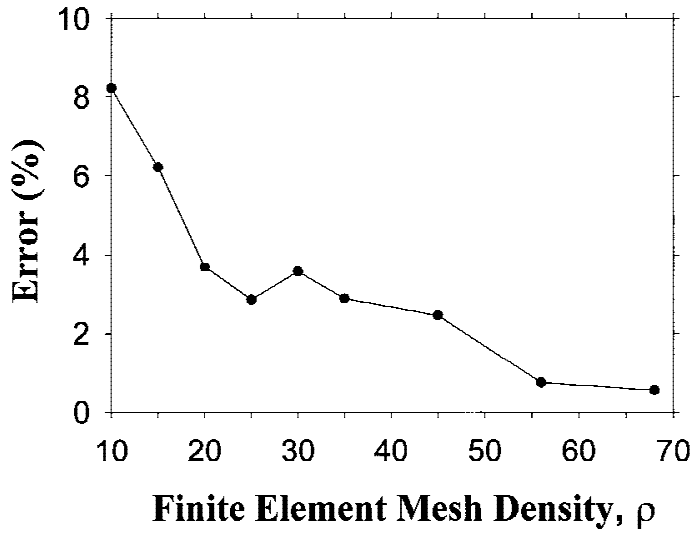

Fig. 8. Percent error in scattering coefficient $S$ for finite-element calculations compared to Mie theory for the case of a 197-nmdiameter, $n=2.74$ sphere in $n=1.514$ resin; the light wavelength is $560 \mathrm{~nm}$.

octant of the particle, which is equivalent to 180 illumination directions over the entire particle. Using the finite-element method, the far-field scattering parameters $S=23.97 \mu^{-1}$ and $\sigma=10.07 \mu \mathrm{m}^{-1}$ have been computed for the rutile particle shown in Fig. 9.

\section{Scattering by Two Interacting, Morphological Rutile Particles}

An issue of great interest in the paint industry is the crowding effect, in which the light-scattering efficiency of a white pigment decreases on a per-pound basis as the pigment volume concentration in a paint film increases. While experimental evidence of the crowding effect is well established, it has not been previously studied on the microstructural level using a rigorous treatment of the near-field electromagnetic interactions between neighboring particles. We have performed finiteelement computations for the case of two morphological rutile titania particles which approach each other in a resin. In this case, two particles with the same geometry and anisotropic optical properties as the particle shown in Fig. 9 are positioned within a finite-element model with interparticle separations ranging from $0.5 \mu \mathrm{m}$ to $0 \mu \mathrm{m}$ (touching). Cross-sectional views of the finite-element models are shown in upper panels of Figs. 10-12, and the logarithms of scattered intensity through the same cross section are shown in the lower panels. In each of these figures, the illumination direction is perpendicular to the plane of the cross sections shown, and the light polarization is parallel to the long axes of the particles. Asymmetry in the near-field scattered intensities around each of the particles is apparent in each of these figures, indicating that the two particles are interacting at these separations.

Using the techniques outlined in Sect. IV, we have simulated diffuse illumination conditions for each of the interparticle separations investigated. The presence of three mutually perpendicular mirror planes in the geometry of the finite-element models limited the number of illumination directions of 25 in one octant of the finite-element model. The computed scattering coefficient $S\left(\mu \mathrm{m}^{-1}\right)$ is shown in Fig. 13. The computed angle-weighted scattering coefficient $\sigma\left(\mu \mathrm{m}^{-1}\right)$ as a function of interparticle separation is shown in Fig. 14.

\section{Discussion}

The finite-element method has been applied to the computation of light-scattering properties of systems with realistic, complex microstructures for which no analytical solutions exist. The morphological rutile particle with anisotropic optical constants is a far more accurate representation of an actual pigment particle than an isotropic sphere. In addition, the com- putations performed for a two-particle system of this type include the effects of near-field optical interactions between particles, which are the basis of the crowding effect in extensive paint films.

\section{(1) Light Scattering by a Single, Morphological Rutile Particle}

It is of interest to compare the far-field scattering parameters $S$ and $\sigma$ computed for the rutile particle to those from Mie theory for the sphere with equivalent volume, using both the average index and weighted sum approximations. The volume of the rutile particle shown in Fig. 9 is $7.77 \times 10^{3} \mu \mathrm{m}^{3}$, corresponding to an equivalent spherical diameter of $0.246 \mu \mathrm{m}$. Such comparisons provide a sense of the adequacy of Mie theory to describe the optics of realistic rutile particles. Table I shows the results of this comparison, with $S$ and $\sigma$ values shown for the finite-element computation for the rutile particle and for the equivalent-volume sphere computed using both the average index and weighted sum approximations. For the average index approximation, the $S$ result is $1.4 \%$ lower than the finite-element result, and the angle-weighted scattering coefficient $\sigma$ result is $4.6 \%$ higher than the finite-element result. For the weighted sum approximation, the $S$ result is $6.4 \%$ lower than the finite-element result, and the angle-weighted scattering coefficient $\sigma$ is in agreement with the finite-element result. For this particular rutile particle, at least, the scattering properties of the equivalent-volume sphere computed using the two approximations and Mie theory are within $\sim 5 \%$ of the finiteelement result.

\section{(2) Crowding Effects in a System of Two Interacting Rutile Particles}

The scattering parameter $S$ has been computed for the case of the two-particle system described in Section IV as a function of the interparticle separation distance. This result is shown in Fig. 13; the horizontal line indicates the value of the scattering coefficient for the isolated particle shown in Fig. 7 for comparison with the two-particle results. The scattering coefficient $S$ decreases with decreasing separation between the two particles. For an interparticle separation of $0.5 \mu \mathrm{m}$, the scattering coefficient is very nearly equal to that associated with the isolated particle. For the case of zero interparticle separation, the value of the scattering coefficient $S$ is $8 \%$ lower than that associated with a single, isolated particle. That is, the twoparticle aggregate scatters $8 \%$ less total light than the isolated particle.

Results for the angle-weighted scattering coefficient $\sigma$ for the two-particle system are shown in Fig. 14 as a function of the interparticle separation distance. In analogy to Fig. 13, the horizontal line in the figure indicates the $\sigma$ value associated with the isolated particle shown in Fig. 7. The results in Fig. 14 show that the value of the angle-weighted scattering coefficient $\sigma$ for the interparticle separation of $0.5 \mu \mathrm{m}$ is nearly equal to that associated with the isolated particle. For the case of the two particles touching, the value is $20 \%$ lower, indicating that the two-particle aggregate is significantly less effective in the limit of multiple scattering than the isolated particle.

The crowding effect is illustrated in Figs. 13 and 14 on the scale of a two-particle system. The results represent a specific case and are not represented as being broadly applicable to extensive paint films, since the effects of factors such as particle size, particle orientation, and the number of interacting particles are not addressed. Instead, these results represent a valuable first step in identifying the factors which are most important in the crowding effect. These results further highlight the applicability of the finite-element method to lightscattering problems which have not been previously addressed using an explicit approach.

\section{(3) Mie Theory in Particle Size Analysis by Light Scattering}

It is common in particle sizing methods based upon light scattering to use Mie theory to compute particle size distribu- 


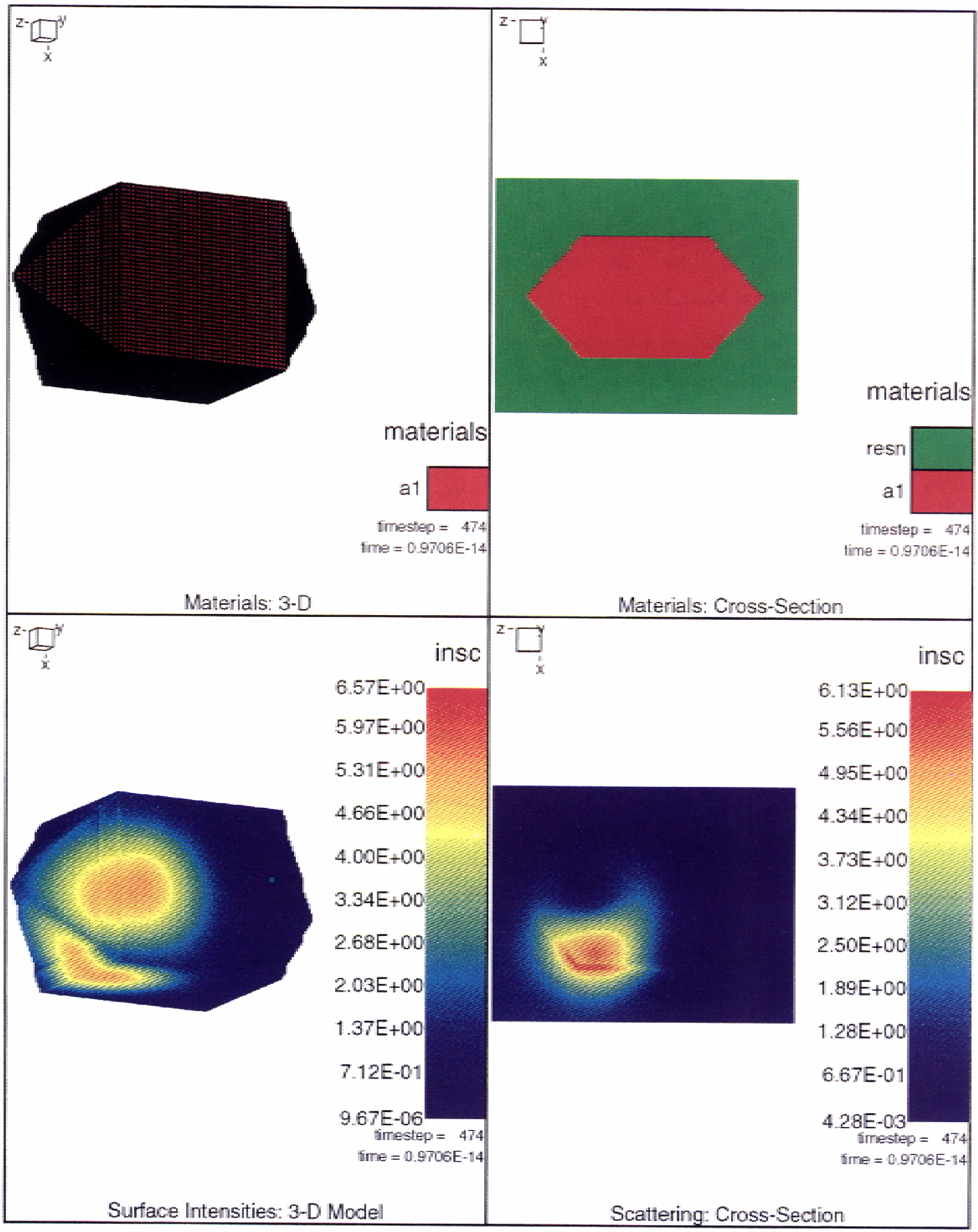

Fig. 9. Finite-element model and near-field scattering results for an anisotropic, morphological rutile particle with a width of $0.175 \mu \mathrm{m}$, and a length of $0.35 \mu \mathrm{m}$ in $n=1.514 \mathrm{resin}$; the light wavelength is $560 \mathrm{~nm}$. On the left are three-dimensional views of the model and scattered light intensity, while on the right are cross-sectional views of the model and scattered light intensity.

tions for particles which are in reality nonspherical. ${ }^{24}$ In these methods, the light-scattering properties of a system are used to determine equivalent spherical particle sizes by fitting scattering data to a size distribution. It is therefore of considerable interest to compare the results of the finite-element calculations on systems containing realistically shaped rutile particles to the results of Mie theory. This can be achieved by back-calculating equivalent spherical diameters corresponding to the morphological rutile particle based upon the scattering parameters $S$ and $\sigma$ rather than upon particle volume (as in Table I).

Using the results, $S=23.97 \mu \mathrm{m}^{-1}$ and $\sigma=10.07 \mu \mathrm{m}^{-1}$ for the isolated rutile particle shown in Fig. 9, the Mie theory 


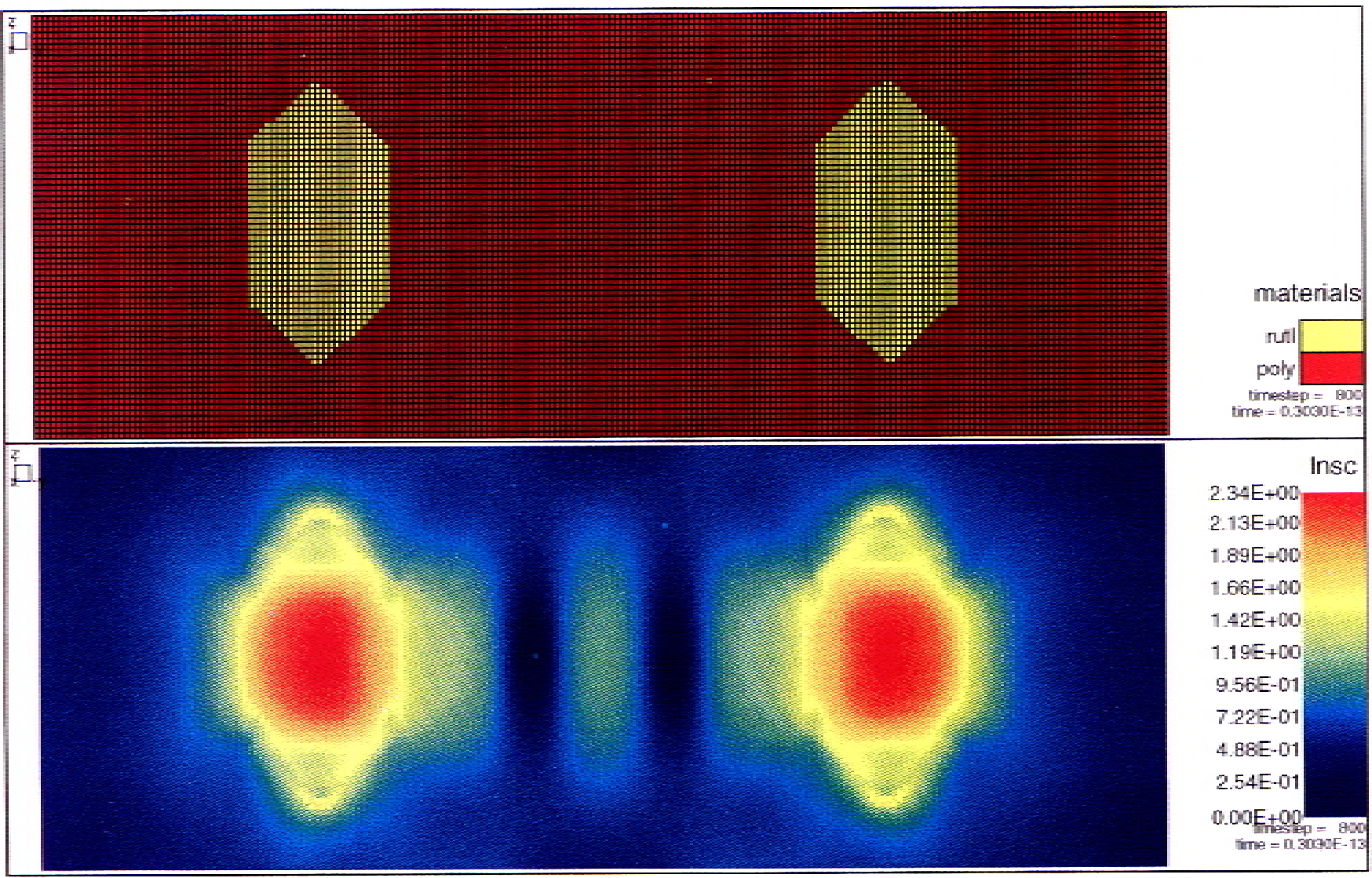

Fig. 10. Finite-element model cross section and near-field scattering results for two anisotropic morphological rutile particles in $n=1.514$ resin for light incident normal to the cross section shown and with a wavelength of $560 \mathrm{~nm}$. The interparticle spacing is $0.5 \mu \mathrm{m}$.

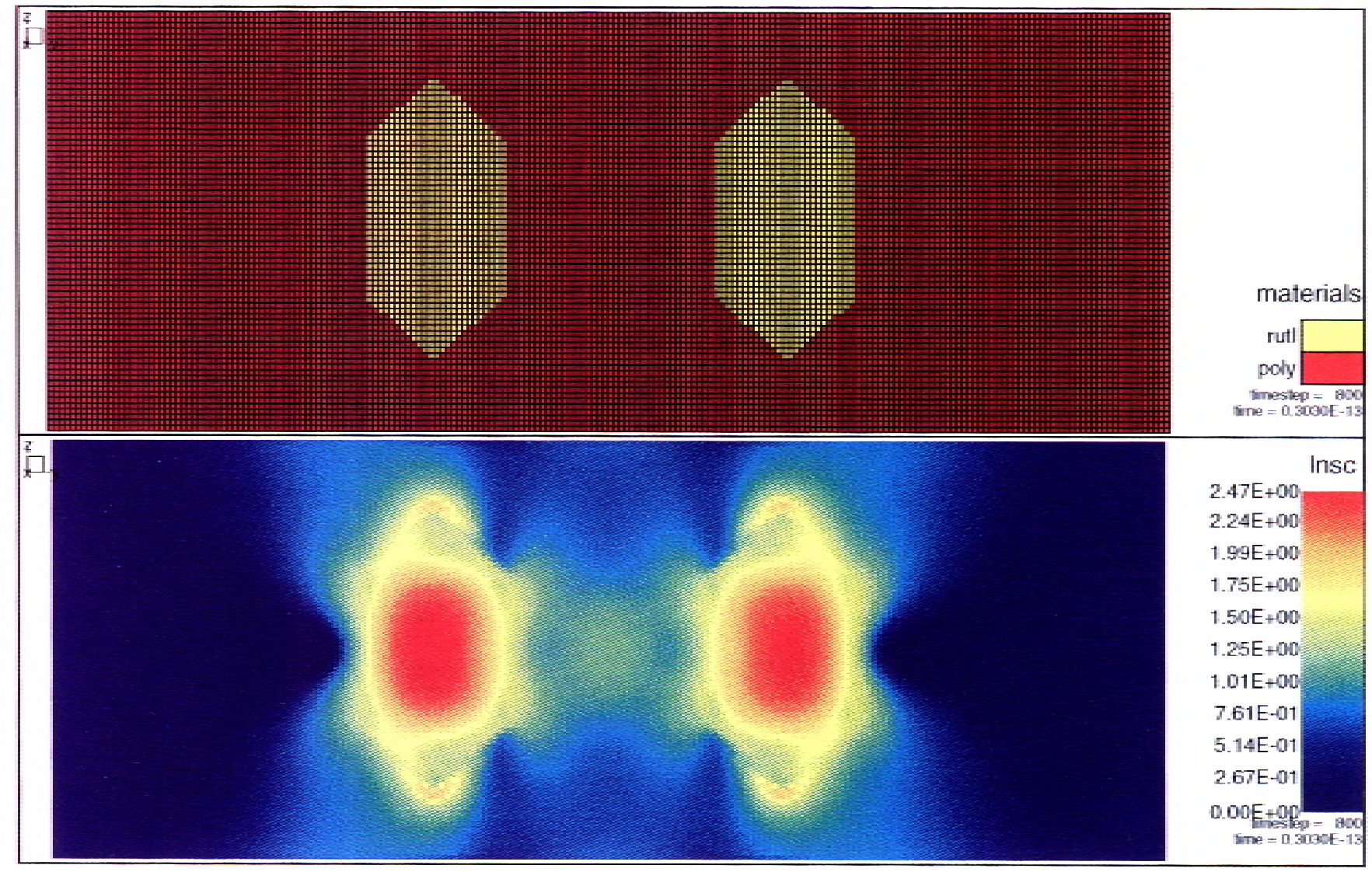

Fig. 11. Finite-element model cross section and near-field scattering results for two anisotropic morphological rutile particles in $n=1.514$ resin for light incident normal to the cross section shown and with a wavelength of $560 \mathrm{~nm}$. The interparticle spacing is $0.2 \mu \mathrm{m}$. 


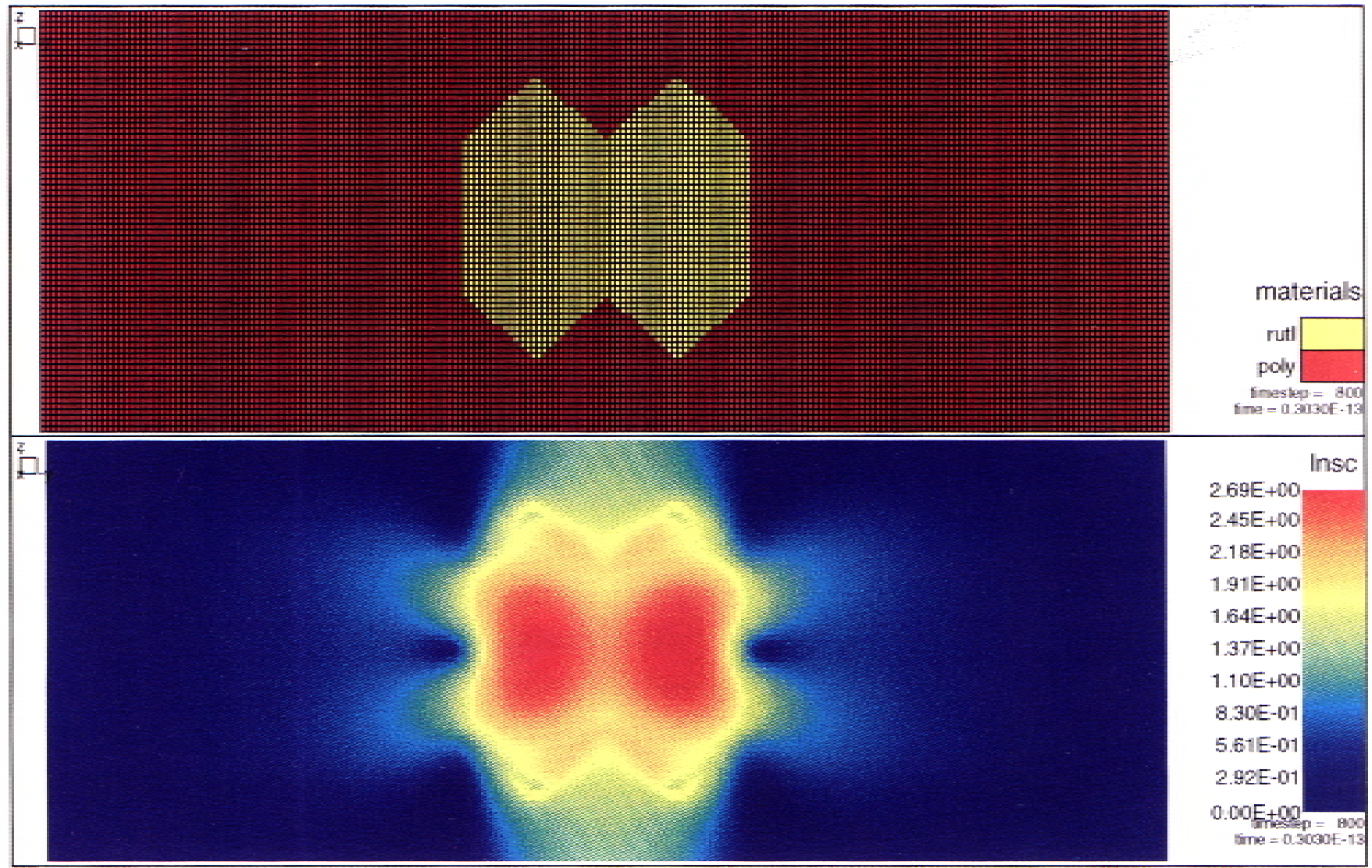

Fig. 12. Finite-element model cross section and near-field scattering results for two anisotropic morphological rutile particles in $n=1.514$ resin for light incident normal to the cross section shown and with a wavelength of $560 \mathrm{~nm}$. The interparticle spacing is $0 \mu \mathrm{m}$.

results shown in Figs. 2-5 have been used to compute the spherical diameters exhibiting these same far-field scattering parameters. These results are shown in Table II. for both the average index and weighted sum approximations, four different spherical diameters correspond to matching $S$ values, and two different spherical diameters correspond to matching angleweighted scattering coefficient $\sigma$ values. The multiple sphere diameters corresponding to the single $S$ and $\sigma$ values associated with this particle result from multiple occurrences of these values in the curves shown in Figs. 2-5. These results highlight the potential ambiguity in using Mie theory to impute equivalent spherical diameters to nonspherical particles based upon far-field scattering properties.

As in the case of the isolated rutile particle, it is possible to

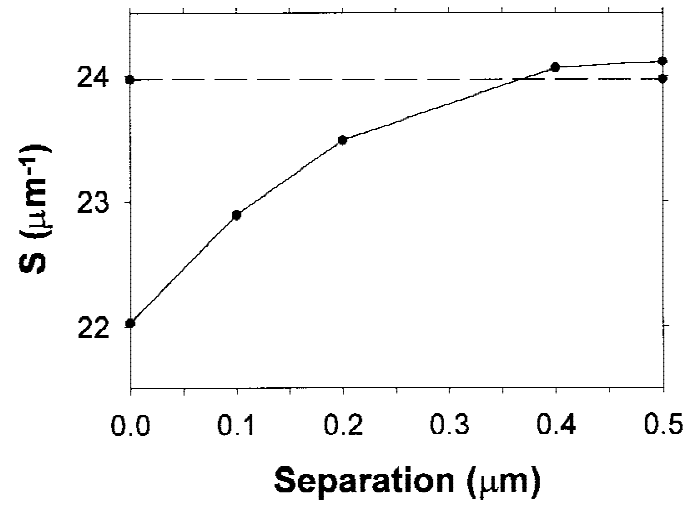

Fig. 13. Scattering coefficient $S$ for two morphological rutile particles as a function of interparticle separation. The horizontal line shows the results for two noninteracting morphological rutile particles.

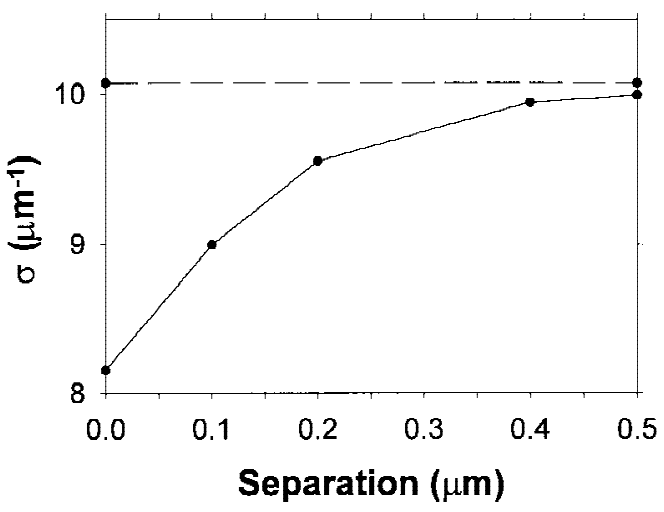

Fig. 14. Angle-weighted scattering coefficient $\sigma$ for two morphological rutile particles as a function of interparticle separation. The horizontal line shows the results for two noninteracting morphological rutile particles.

Table I. Comparison between the Far-Field Scattering Coefficients for the Single Rutile Particle and the Equivalent Volume Sphere, Using Both the Average Index and Weighted Sum Approximations

\begin{tabular}{lcc}
\hline & $\begin{array}{c}\text { Scattering } \\
\text { coefficient } \\
S\left(\mu \mathrm{m}^{-1}\right)\end{array}$ & $\begin{array}{c}\text { Angle-weighted } \\
\begin{array}{c}\text { scattering coefficient } \\
\sigma\left(\mu \mathrm{m}^{-1}\right)\end{array}\end{array}$ \\
\hline $\begin{array}{l}\text { Anisotropic rutile particle } \\
(W=0.175 \mu \mathrm{m}, \text { aspect ratio } 2)\end{array}$ & 23.97 & 10.07 \\
$\begin{array}{l}\text { Equivalent-volume sphere } \\
\quad(n=2.74)\end{array}$ & 22.44 & 10.07 \\
$\begin{array}{l}\text { Equivalent-volume sphere } \\
\quad(\text { weighted sum of } n=2.64\end{array}$ & & \\
$\quad$ and $n=2.94$ results $)$ & 23.64 & 10.54 \\
\hline
\end{tabular}


Table II. Diameters of Spheres Exhibiting the Same Far-Field Scattering Coefficients as the Single Rutile Particle, Using Both the Average Index and Weighted Sum Approximations

\begin{tabular}{lcr}
\hline & $\begin{array}{c}\text { Scattering } \\
\text { coefficient } \\
S \text { basis }(\mu \mathrm{m})\end{array}$ & $\begin{array}{c}\text { Angle-weighted } \\
\text { scattering } \\
\text { coefficient } \\
\sigma \text { basis }(\mu \mathrm{m})\end{array}$ \\
\hline $\begin{array}{c}\text { Equivalent spherical diameter } \\
\text { (based upon } n=2.74)\end{array}$ & $\begin{array}{c}0.186 \\
\text { or } 0.212 \\
\text { or } 0.254\end{array}$ & $\begin{array}{r}0.183 \\
\text { or } 0.247\end{array}$ \\
$\begin{array}{c}\text { Equivalent spherical diameter } \\
\text { (based upon weighted sum of } \\
n=2.64 \text { and } n=2.94 \text { results) }\end{array}$ & $\begin{array}{l}\text { or } 0.204 \\
\text { or } 0.248\end{array}$ & $\begin{array}{c}0.179 \\
\text { or } 0.287\end{array}$ \\
\hline
\end{tabular}

Table III. Diameters of Spheres Exhibiting the Same Far-Field Scattering Coefficients as the System of Two Rutile Particles for Each Interparticle Separation Distance Considered, Using Both the Average Index and Weighted Sum Approximations

\begin{tabular}{ccc}
\hline $\begin{array}{c}\text { Interparticle } \\
\text { separation }(\mu \mathrm{m})\end{array}$ & $\begin{array}{c}\text { Scattering coefficient } \\
S \text { basis }(\mu \mathrm{m})\end{array}$ & $\begin{array}{c}\text { Angle-weighted } \\
\text { scattering coefficient } \\
\sigma \text { basis }(\mu \mathrm{m})\end{array}$ \\
\hline 0.5 & 0.186 & 0.183 \\
& 0.211 & 0.278 \\
0.254 & \\
0.4 & 0.292 & \\
& 0.186 & 0.183 \\
& 0.212 & 0.279 \\
0.2 & 0.254 & \\
& 0.293 & 0.181 \\
& 0.184 & 0.285 \\
0.1 & 0.215 & 0.337 \\
& 0.252 & 0.349 \\
& 0.295 & 0.178 \\
0 & 0.182 & 0.295 \\
& 0.22 & 0.328 \\
& 0.249 & 0.354 \\
& 0.297 & 0.171 \\
& 0.180 & 0.360 \\
\hline
\end{tabular}

determine the diameters of spheres with equivalent far-field scattering properties for the case of two interacting rutile particles described above. This case is especially interesting, since the total volume of scattering material remains constant as the two rutile particles approach each other, yet the farfield scattering properties of the system change markedly as the interparticle distance changes. These results are presented in Table III, with the sphere diameters producing the same values of the scattering coefficients $S$ and $\sigma$ as the two-particle system for each of the interparticle separation distances considered. As in the case of the single rutile particle, multiple values of $S$ and $s$ occur for each of the interparticle separation distances.

\section{Conclusions}

We utilize a finite-element method which produces rigorous solutions to Maxwell's equations to determine computationally the light-scattering properties of complex particulate microstructures. In the present study, we use the finite-element method first to compute the light-scattering properties of a single, morphological rutile particle with a representative size and shape. These results are compared to the light-scattering properties of the optically isotropic, equivalent volume sphere using Mie theory. Neither the average index nor the weighted sum approximation offers clear advantages in this case. Second, the far-field light-scattering properties of two such particles interacting at near field are determined as a function of the interparticle separation. The agglomerated pair of particles exhibits a $20 \%$ decrease in the scattering parameter associated with the hiding power of a paint film relative to the single particle. The basis for this decrease is the same as for the crowding effect observed in extensive paint films. The results of both sets of computations are compared to Mie theory to determine the sizes of spherical particles with equivalent scattering cross sections. These comparisons highlight the inherent difficulties in using Mie theory to evaluate particle size by light-scattering methods.

Acknowledgments: The authors acknowledge T. M. Spitler for helpful discussions about this work and J. Mould Jr. for computational support in implementing the finite-element code. The authors also thank E. D. Boyes for providing the micrograph in Fig. 1.

\section{References}

${ }^{1} \mathrm{H}$. Braun, A. Baidins, and R. E. Marganski, “TiO 2 Pigment Technology: A Review," Prog. Organic Coat., 20, 105-38 (1992).

${ }^{2}$ T. C. Patton, Pigment Handbook. Wiley, New York, 1973

${ }^{3}$ D. P. Fields, R. J. Buchacek, and J. G. Dickinson, "Maximum $\mathrm{TiO}_{2}$ Hiding Power-The Challenge," J. Oil Colour Chem. Assoc., 2, 87-93 (1993).

4J. G. Balfour, "Opacity and Titanium Dioxide Pigments," J. Oil Colour Chem. Assoc., 73, 225 (1990).

${ }^{5} \mathrm{G}$. Mie, "Beitrage zur Optik Truber Medien, Speziell Kolloidaler Metallosungen," Ann. Phys., 25, 377-55 (1908).

${ }^{6}$ H. C. van de Hulst, Light Scattering by Small Particles. Dover, New York, 1981

${ }^{7}$ C. F. Bohren and D. R. Huffman, Absorption and Scattering of Light by Small Particles. Wiley-Interscience, New York, 1983.

${ }^{8}$ W. D. Ross, "Theoretical Computation of Light Scattering Power: Comparison Between $\mathrm{TiO}_{2}$ and Air Bubbles," J. Paint Technol., 43, 50-66 (1971).

${ }^{9}$ S. Fitzwater and J. W. Hook III, "Dependent Scattering Theory: A New Approach to Predicting Scattering in Paints,' J. Coat. Technol., 57, 39-47 (1985).

${ }^{10}$ R. J. Bruehlman, L. W. Thomas, and E. Gonick, "Effect of Particle Size and Pigment Volume Concentration on Hiding Power of Titanium Dioxide", Proceedings of the Fifth Biennial Spring Symposium of the Pacific Societies for Paint Technology, Feb. 25, 1960. Reprinted from Official Digest, February 1961

${ }^{11}$ D. F. Tunstall and M. J. Hird, "Effect of Particle Crowding on Scattering Power of $\mathrm{TiO}_{2}$ Pigments," J. Paint Technol., 46, 33-40 (1974).

${ }^{12}$ J. H. Braun, "Crowding and Spacing of Titanium Dioxide Pigments," J. Coat. Technol., 60, 67-71 (1988).

${ }^{13}$ B. R. Palmer, P. Stamatakis, C. F. Bohren, and G. C. Salzman, “A Multiple Scattering Model for Opacifying Particles in Polymer Films," J. Coat. Technol., 61, 41-47 (1989).

${ }^{14}$ J. L. Volakis, A. Chatterjee, and L. C. Kempel, "Review of the Finite Element Method for Three-Dimensional Electromagnetic Scattering," J. Opt. Soc. Am. A, 11, 1422-33 (1994).

${ }^{15}$ G. L. Wojcik, "Theoretical Description of the EMFlex Finite Element Formulation," unpublished work, 1991.

${ }^{16}$ G. L. Wojcik, D. K. Vaughan, and L. K. Galbraith, "CCalculation of Scatter from Structures on Silicon Surfaces," Proc. SPIE-Int. Sec. Opt. Eng., 774, 21-31 (1987).

${ }^{17}$ G. L. Wojcik, J. Mould, and L. West, “"Time-Domain Finite Element Modeling of 3D Integrated Optical Devices," Integr. Photon. Res. Tech. Digest, 10 [March] 112-15 (1993).

${ }^{18}$ A. D. Chaudari, Y. Lu, L. C. West, and C. Roberts, "Highly Compact Optical Waveguides with a Novel Pedestal Geometry," IEEE Photon. Technol. Lett., 7, 526-28 (1995).

${ }^{19}$ L. C. West, C. Roberts, J. Dunkel, G. Wojcik, and J. Mould, "Non Uniform Grating Couplers for Coupling of Gaussian Beams to Compact Waveguides," Integr. Photon. Res. Tech. Dig., (1994).

${ }^{20}$ L. C. West, C. Roberts, and E. C. Piscani, "Ultra High Confinement Waveguides for Very Large Scale Integrated Optics (VLSOI) with ThreeDimensional Packaging,"' Integr. Photon. Res. Tech. Dig., (1996).

${ }^{21}$ R. F. Harrington, Time-Harmonic Electromagnetic Fields. McGraw-Hill, New York, 1961.

${ }^{22}$ C. F. Bohren, "Multiple Scattering of Light and Some of its Observable Consequences," Am. J. Phys., 55, 524-33 (1987).

${ }^{23}$ M. W. Ribarsky, Handbook of Optical Constants (E. D. Palik, ed.) Academic Press, New York, 1985.

${ }^{24}$ T. Allen, Particle Size Measurement. Chapman and Hall, London, U.K., 1990. 